\title{
Inhibition of the IncRNA Mirt1 Attenuates Acute Myocardial Infarction by Suppressing NF-kB Activation
}

\author{
Xiangrao Lia Jian Zhou ${ }^{b}$ Kai Huang ${ }^{a}$ \\ aDepartment of Cardiovascular Diseases, Union Hospital, Tongji Medical College, Huazhong University \\ of Science and Technology, Wuhan, 'Department of Cardiovascular Diseases, Xinyang Central Hospital, \\ Xinyang, China
}

\section{Key Words}

Long noncoding RNA Mirt1 - Acute myocardial infarction - Inflammation - Cardiomyocyte apoptosis $・ N F-K B$

\begin{abstract}
Background/Aims: The expression of a novel IncRNA, myocardial infarction associated transcript 1(Mirt1), has been shown to be upregulated in acute myocardial infarction (AMI). However, the role of Mirt1 in AMI is not clear. Methods: In this study, we analyzed the level of Mirt1 in cardiomyocytes and cardiac fibroblasts in AMI mice. Moreover, adenovirus mediated knockdown of Mirt1 was employed to clarify its roles in AMI mice or cultured cardiac fibroblasts. The cardiac functions and infarct size of AMI mice were examined, and tissues and cultured cells were collected and processed for histology and biochemical examination. Results: We demonstrated that Mirt1 was mainly expressed in cardiac fibroblasts, and that knockdown of Mirt1 improved cardiac functions, decreased cardiomyocytes apoptosis and attenuated inflammatory cell infiltration in vivo. Furthermore, knockdown of Mirt1 in cardiac fibroblasts not only attenuated the apoptosis of cardiomyocytes, but also suppressed the migration of macrophages under hypoxia in vitro. NF-KB signaling pathway, activated under hypoxia, was also inhibited by Mirt1 knockdown in fibroblasts. Conclusions: Knockdown of Mirt1 attenuates AMI injury presumably by decreasing cardiomyocytes apoptosis and reducing inflammatory cell infiltration. These effects could be attributed, at least partly, to inhibition of the NF-KB pathway, resulting in decreased expression of inflammatory factors.

\section{Introduction}

AMI remains the most serious cardiac event with high morbidity and mortality, in spite of declining mortality over the past three decades [1]. Inflammatory response and cardiomyocyte apoptosis are the main characteristics of AMI and play an important role in impaired myocardial function and heart failure [2,3]. Therefore, therapeutic approaches

X. Li and J. Zhou contributed equally to this work. 
Li/Zhou/Huang: Inhibition of Mirt1 Attenuates Acute Myocardial Infarction

targeting the molecular mechanisms involved in the inflammatory response and in cardiomyocyte apoptosis will provide novel treatments for AMI.

Several studies have suggested that cardiac fibroblasts (CFs) might contribute to the initiation of the inflammatory reaction in the acute stage during myocardial infarction [4]. Activation of the inflammatory cascade in CFs has been demonstrated to play a pivotal role in AMI [4]. It has been proven that the levels of cytokines, such as IL-1 $\beta$, IL- 6 and other factors secreted by CFs, might contribute to pathological cardiac processes $[5,6]$. CFs, which are sensitive to AMI injury, might be a critical mediator of the inflammatory response in AMI injury.

Long noncodingRNA (lncRNA), consisting of RNA molecules of longer than 200 nucleotides, make up a novel class of noncoding RNA. There is increasing evidence that lncRNAs play important roles in regulation of physical and pathological processes in many organisms, including in the cardiovascular system [7, 8]. For example, the lncRNA H19 is upregulated during cardiomyocyte hypertrophy [9], and IncRNA HOTAIR is associated with cardiac functions in sepsis [10]. LncRNA Mirt1, which was first identified by Zangrando et al., is significantly upregulated during AMI [11]. However, the effects of Mirt1 in AMI remain unclear. In the present study, we demonstrated that inhibiting the expression of Mirt1 in CFs could reduce the inflammatory response and ameliorate cardiomyocyte apoptosis in vivo and in vitro.

\section{Material and Methods}

Animals

Male C57BL/6J mice aged 8-10 weeks were purchased from Beijing HFK Bioscience Co., Ltd. (Beijing, China). Feed and water were provided ad libitum, while light was provided 12 hours a day during the entire experimental period. All animal studies were performed in adherence with the Guide for the Care and Use of Laboratory Animals published by the US National Institutes of Health and prior approval was obtained from the Animal Care and Utilization Committee of Huazhong University of Science and Technology.

AMI in mice

Acute myocardial infarction was induced by permanent left-anterior descending coronary artery ligation as previously described $[12,13]$. Briefly, mice were anaesthetized by using pentobarbital sodium (50 mg/kg, P3761, Sigma-Aldrich, St Louis, MO, USA) by intraperitoneal injection. Mice were ventilated with room air using a rodent ventilator after oral intubation, and subjected to a left-sided thoracotomy in the supine position. The left-anterior descending coronary artery (LAD) was visualized and ligated using a 6-0 prolene suture under a stereomicroscope. The distal end of myocardial discoloration was observed under the microscope to confirm regional ischemia. Sham operated mice underwent the same procedure without ligation of the LAD. Mice were divided into four groups: Sham+Ad-shCon, Sham+Ad-shMirt1, AMI+Ad-shCon, and AMI+Ad-shMirt1. Adenovirus were administered by intramyocardial injection 7 days before surgery (Fig. 1C). Three days after operation, echocardiography and hemodynamic measurements were performed before mice were sacrificed. Then the hearts were harvested and fixed in 4\% paraformaldehyde overnight for histological staining, and the fresh infarcted areas of myocardium were collected for quantitative realtime PCR and western blotting. Samples from the sham group were collected as control.

\section{Echocardiography and hemodynamic analysis}

Cardiac functions were assessed by two-dimensional echocardiograph using a Visual Sonics Vevo 2100 Ultrasound machine (VisualSonics Inc., Toronto, Ontario, Canada) equipped with a 30-MHz probe as described in our previous study [14]. Mice were anesthetized with $1.5 \%$ isoflurane, and subjected to echocardiography both pre-surgery and post-MI. Recording was performed as described previously [14]. Relevant cardiovascular parameters were calculated as described previously [15]. Hemodynamic measurements were taken using a Millar SPR-1000 catheter. Cardiovascular factors were calculated off-line using LabChart (ADInstruments, Bella Vista, NSW, Australia). Measurement procedures were performed as described previously [16]. 


\section{Cellular Physiology Cell Physiol Biochem 2017;42:1153-1164 \begin{tabular}{ll|l} 
and Biochemistry Published onlIne: June30, 2017 & $\begin{array}{l}\text { (c) } 2017 \text { The Author(s). Published by S. Karger AG, Basel } \\
\text { www.karger.com/cpb }\end{array}$
\end{tabular}}

\section{Isolation of cardiac fibroblasts and cardiomyocytes from adult mice}

Adult mouse CFs and cardiomyocytes were isolated from male C57BL/6J aged 8 to 10 weeks as previously described $[17,18]$. In brief, mice were sacrificed, and hearts were harvested, perfused and digested with collagenase II. Then, cells including cardiomyocytes and non-cardiomyocytes dissociated from the heart were separated by gravity sedimentation. The precipitate, composed mainly of cardiomyocytes, and the supernatant, almost all non-cardiomyocytes, were collected separately. The non-cardiomyocytes were then cultured in DMEM with $10 \% \mathrm{FBS}$ at $37^{\circ} \mathrm{C}$ with $5 \% \mathrm{CO}_{2}$ for 2 hour, then the medium was replaced with fresh medium to continue the culture. The cells remaining on the plate were mostly cardiac fibroblasts.

\section{Isolation of neonatal mouse cardiomyocytes and fibroblasts}

Primary cultures of neonatal mouse cardiomyocytes were generated as described previously [19]. Briefly, hearts collected from 1-2-day-old mice were washed, minced into small pieces(approximately 0.5$\left.1 \mathrm{~mm}^{3}\right)$ and predigested in isolation medium $(0.5 \mathrm{mg} / \mathrm{ml}$ trypsin-HBSS solution) under gentle agitation overnight at $4^{\circ} \mathrm{C}$. Cardiac cells were subjected to collagenase type II (240 units/mL) (Worthington Biochemical, Lakewood, USA) in HBSS for $10 \mathrm{~min}$ at $37^{\circ} \mathrm{C}$, and the supernatant was collected. All the supernatant was passed through a $100-\mu \mathrm{m}$ filter, then centrifuged for $5 \mathrm{~min}$ at $100 \times \mathrm{g}$. The precipitate was re-suspended in DMEM/F12 (1:1) medium supplemented with 10\% fetal calf serum and 1\% penicillinstreptomycin, plated into $10 \mathrm{~cm}$ cell culture dishes and incubated for 2 hours at $37^{\circ} \mathrm{C}$ with $5 \% \mathrm{CO}_{2}$. Then, non-adherent cardiomyocytes were gathered and plated into new dishes, and the remaining cells adhering on the dishes were mostly cardiac fibroblasts. Cardiomyocytes and fibroblasts were cultured in DMEM/F12 (1:1) medium for 48 hours, and then were used for subsequent experiments. For hypoxia experiments, cells were cultured under hypoxic conditions in a tri-gas incubator with $1 \% \mathrm{O}_{2}, 5 \% \mathrm{CO}_{2}$, and $94 \% \mathrm{~N}_{2}$.

\section{Isolation of peritoneal macrophages and migration assay}

Peritoneal macrophages were harvested from C57BL/6 mice (8 - 10 weeks old) as described [20]. Aliquots of cell suspension containing $1 \times 10^{6}$ macrophages were added into the upper chamber of Transwell chambers ( $8 \mu \mathrm{m}$ pore size membrane, Corning, NY, USA) in medium without serum. Conditioned medium collected from the fibroblasts cultured under hypoxic conditions and infected with Ad-shMirt1 or Ad-shCon was added into the lower chamber. After 16 hours, the migrated macrophages were fixed in 4\% paraformaldehyde, and stained with crystal violet. Cells remaining on the upper side of the membrane were removed by gently swiping with a cotton buds. The mean number of migrated cells was quantified in three randomly-selected fields using a microscope.

\section{Recombinant virus vector construction and infection}

Adenovirus carrying shRNA against the mouse lncRNA Mirt1 or control shRNA vectors were purchased from Shanghai OOBIO Biotechnology Company (Shanghai, China). Mice were administrated with $1 \times 10^{8}$ pfu purified adenovirus by direct intramyocardial injection as reported [21] 7 days before surgery. Cells were infected with adenovirus at a multiplicity of infection (MOI) of 50.

\section{Measurement of area at risk and infarct size}

Three days afer AMI, Evans blue (Sigma-Aldrich) was injected from the cardiac apex of AMI mice to define the nonischemic tissue after anesthesia and thoracotomy. After the hearts were excised and rapidly frozen at $-20^{\circ} \mathrm{C}$, they were cut into $2 \mathrm{~mm}$ thick slices. The slices were incubated in $1 \%$ TTC(2, 3, 5-triphenyl tetrazolium chloride, Sigma-Aldrich) in PBS(phosphate-buffered saline, $\mathrm{pH} \mathrm{7.4)} \mathrm{for} 30$ min at $37{ }^{\circ} \mathrm{C}$ to determine the infarct size, then fixed in $4 \%$ formaldehyde for 2 hours. The slices were photographed and areas at risk and infarct size were measured by Image-Pro Plus 6.0 (Media Cybernetics, Rockville, MD USA).

\section{TUNEL assay}

Terminal deoxynucleotidyl-transferase mediated dUTP nick-end labeling (TUNEL) staining was used to determine the apoptosis level of cardiomyocytes in heart sections. According to the instructions supplied with the in situ cell death detection kit (Roche, Basel, Switzerland), cells cultured on coverslips or frozen tissue sections were fixed in 4\% paraformaldehyde for 1 hour and permeabilized with $0.1 \%$ Triton-X100 for 2 minutes, and then incubated with the TUNEL reaction mixture for 1 hour to stain apoptotic cells. All the nuclei were counter-stained with DAPI. Three random fields in each sample were observed under a 


\section{Cellular Physiology Cell Physiol Biochem 2017;42:1153-1164 \\ and Biochemistry Publis.1159/000478870 $2017 \quad \begin{aligned} & \text { DOI: } 2017 \text { The Author(s). Published by S. Karger AG, Basel } \\ & \text { www.karger.com/cpb }\end{aligned}$}

Li/Zhou/Huang: Inhibition of Mirt1 Attenuates Acute Myocardial Infarction

microscope, and the number of positive apoptotic cells as a percentage of the total number of nuclei was calculated.

\section{Quantitative real-time PCR}

Briefly, after treatment, total RNA from the cells or tissues was extracted using TRIzol reagent (Invitrogen; Thermo Fisher Scientific, Waltham, MA, USA), and reverse transcribed into cDNA using the PrimeScript RT reagent kit (Takara Bio Inc. Shiga, Japan). The mRNA expression of target genes was quantified by use of SYBR Green PCR Master Mix (Takara Bio Inc) with the ABI StepOnePlus Real-Time PCR system (Applied Biosystems, Foster City, CA,USA), and analyzed by normalizing to $\beta$-actin levels and using the comparative $\mathrm{Ct}$ method formula $2^{-\Delta \Delta \mathrm{Ct}}$. Each reaction was performed in duplicate. Primers were as follows: $\beta$-actin forward, GGCTGTATTCCCCTCCATCG; $\beta$-actin reverse: CCAGTTGGTAACAATGCCATGT; Mirt1 forward, CTTGTTGTCTAAGTGAGTA; Mirt1 reverse: TTAATCGTTCCTCTAGTTG; TNF- $\alpha$ forward, GTGGAACTGGCAGAAGAGGC; TNF- $\alpha$ reverse, AGACAGAAGAGCGTGGTGGC; IL-1 $\beta$ forward, GGAAGATTCTGAAGAAGAGACGG; IL-1 $\beta$ reverse, TGAGATTTTTAGAGTAACAGG; IL-6 forward, TAGTCCTTCCTACCCCAATTTCC; IL-6 reverse, TTGGTCCTTAGCCACTCCTTC.

\section{Western blot assay}

Protein extracted from the cardiac tissue of mice or from cultured cells was extracted in RIPA buffer containing protease inhibitor, and protein concentrations were measured using a BCA Protein Assay Kit (Thermo Fisher Scientific) according to the manufacturer's instructions. Equal amounts of denatured protein samples $(60 \mu \mathrm{g})$ were separated by sodium dodecyl sulfate polyacrylamide gel electrophoresis(SDSPAGE), and were transferred to PVDF (polyvinylidene difluoride) membranes (Merck-Millipore, Darmstadt, Germany). Proteins on the membranes were incubated with primary antibodies as follows: Cleaved Caspase-3 antibody (9654S), Bcl-2 antibody (3498S), Bax antibody (2772S) (all from Cell Signaling Technology, Danvers, MA, USA) at $4^{\circ} \mathrm{C}$ overnight, and next day the membranes were treated with ECL reagents (1705061, Bio-Rad, Hercules, CA, USA) prior to visualization using a Fluor Chem E imager(Protein Simple, San Jose, CA, USA) after incubation with HRP- conjugated secondary antibodies (Tianjin Sungene Biotech Co. Ltd., Tianjin, China). The expression levels of specific protein were normalized to levels of $\beta$-tubulin (Tianjin Sungene Biotech).

\section{Histological and immunohistochemical staining}

Three days after surgery, the mouse hearts were harvested and fixed in $4 \%$ paraformaldehyde overnight, then embedded in paraffin. Then the 5 - $\mu \mathrm{m}$ thick sections were cut and processed and treated with a hydrogen peroxide block for 15 - $20 \mathrm{~min}$ to inhibit endogenous peroxidase activity. The sections were then incubated with primary anti-Ly6G or anti- F4/80 antibodies overnight at $4^{\circ} \mathrm{C}$. After washing in PBS three times, the slices were incubated for $30 \mathrm{~min}$ with horseradish peroxidase-conjugated secondary antibody at room temperature. The sections were incubated with DAB chromogen to enable visualization of antigen-antibody complexes to be observed, and were ultimately counterstained with Mayer's hematoxylin to show the nuclei. Three random fields of each sample were photographed under a microscope, and the average positive cell number was used for comparative analysis.

\section{Measurement of inflammatory cytokines}

The level of IL-1 $\beta$ was determined by a mouse ELISA kit (R\&D Systems, Minneapolis, MN, USA) according to the manufacturer's instructions. The level of IL- 6 and tumor necrosis factor- $\alpha$ (TNF- $\alpha$ ) were assessed using the CBA Mouse Inflammation Kit (BD Biosciences, Franklin Lakes, NJ, USA) according to the manufacturer's instructions.

\section{Statistical analysis}

Sample size was determined by power analysis using preliminary data obtained in our laboratory with the following assumptions: $\alpha$ of 0.05 (two-tailed), power of $90 \%$. Data are presented as mean \pm SEM of at least three independent experiments. The differences in the data were analyzed by unpaired, twotailed Student's t-test for two groups or one-way or two-way analysis of variance (ANOVA) for multiple comparisons, followed by a post hoc Student-Newmann-Keuls test. SPSS 13.0 (SPSS Inc., Chicago, IL, USA) was used for statistical analyses, and statistical significance was specified as $P<0.05$. 


\section{Results}

\section{Dynamic changes of Mirt1 in the myocardium of AMI mice}

To quantify the time-dependent change of Mirt1 in the infarcted myocardium of mice, we examined the expression of Mirt1 at 0, 2, 6, 12, 24 and 48 hours after AMI. As shown in Fig. 1A, real-time PCR revealed that the expression of Mirt1 was significantly increased at 6 hours after AMI, when it was almost 3 -fold higher than at 0 hours, and peaked at 24 hours, when it was approximately 10 -fold higher than at 0 hours. The level of Mirt1 decreased to near- normal levels at 48 hours after operation.

Fig. 1. Enhanced expression of Mirt1 after AMI. (A) The levels of Mirt1 at 0,2 , 6, 12, 24 and 48 hours after myocardial infarction. $\mathrm{n}=8$. (B) The levels of Mirt1 in cardiomyocytes and cardiac fibroblasts of mice at 24 hours after myocardial infarction; $\mathrm{n}$ $=10$. (C) Experimental procedure. ${ }^{*} P<0.05,{ }^{* *} P<0.01$.
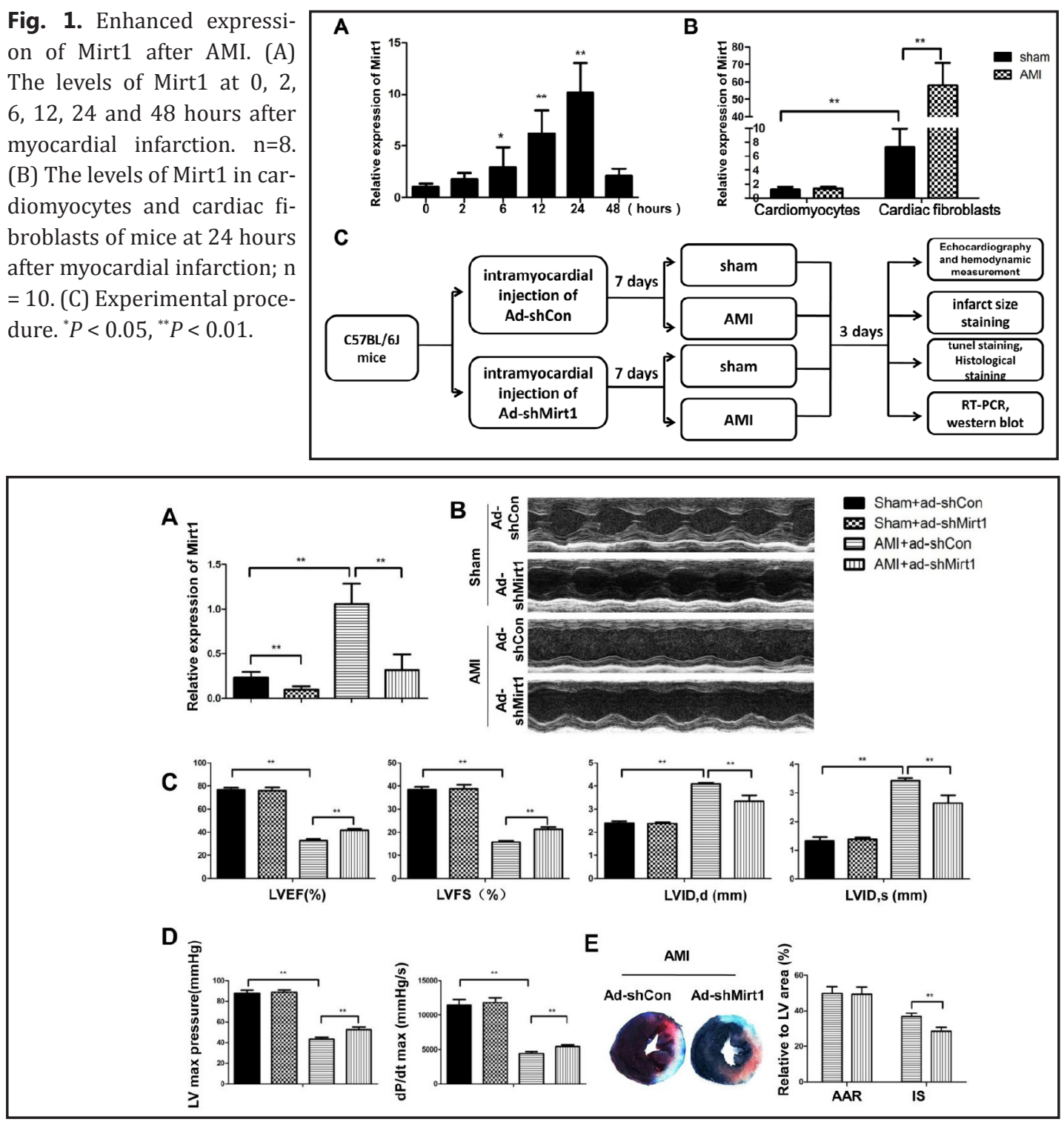

Fig. 2. Knockdown of Mirt1 improves heart functions and decreases infarct size. (A) Expression of Mirt1 in the myocardium of sham and AMI mice treated with Ad-shCon or Ad-shMirt1; $\mathrm{n}=8$. (B) Representative M-mode echocardiography image of the left ventricle (LV) from the four groups at 3 days after AMI; $\mathrm{n}=8$. (C) LVEF, LVFS, LVID.d and LVID.s of mice from the four groups at 3 days after AMI. $n=8$. (D) Maximum LV pressure and $\mathrm{dp} / \mathrm{dt}_{\max }$ parameters of mice from the four groups at 3 days after AMI; $\mathrm{n}=9$. (E) Representative images of Evans blue and TTC staining of myocardium slices and calculation of area at risk (AAR) and infarct size (IS) (relative to LV area) at 3 days after AMI; $\mathrm{n}=10 .{ }^{* *} P<0.01$.

\section{KARGER}




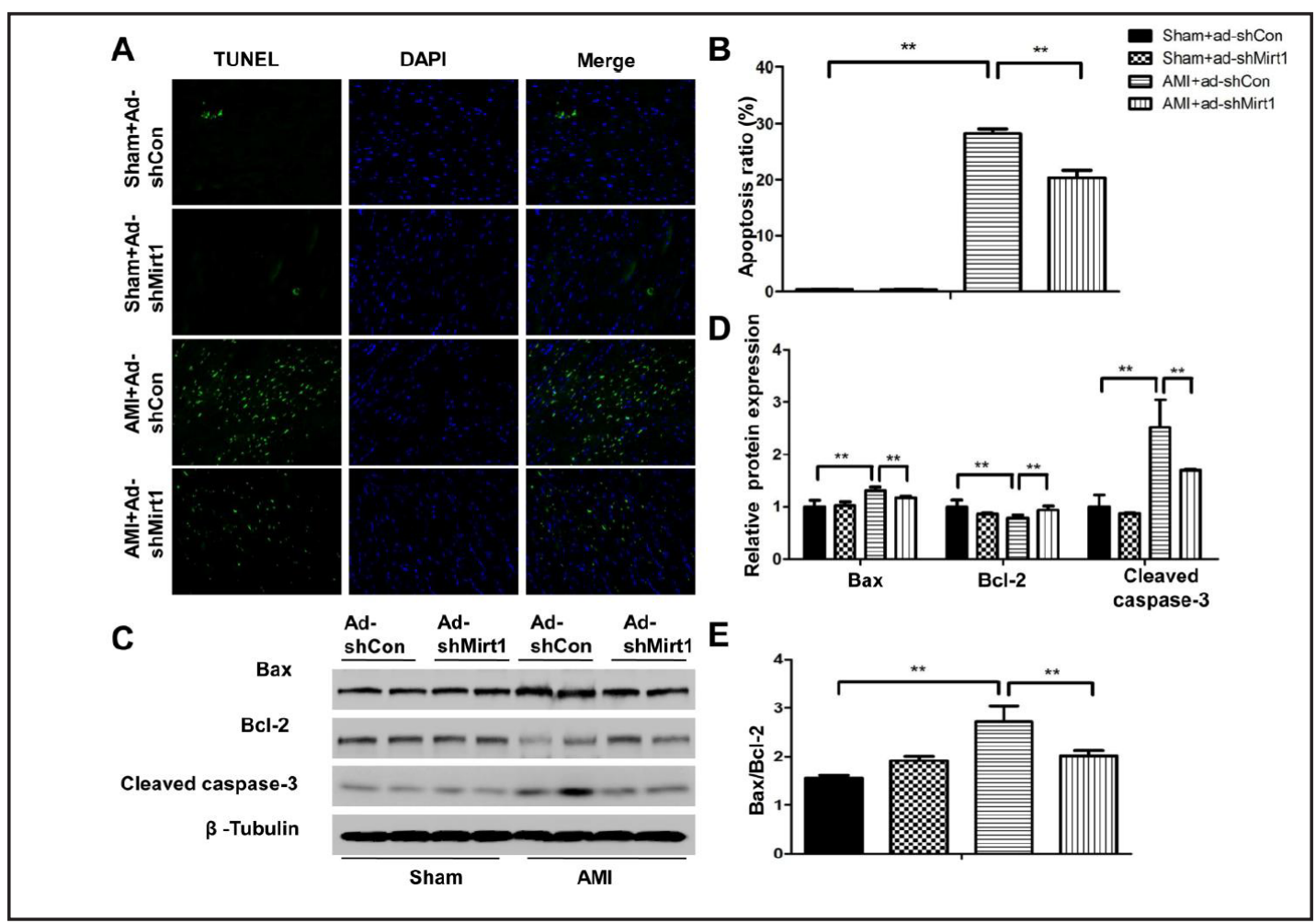

Fig. 3. Knockdown of Mirt1 inhibits apoptosis in vivo. (A) Representative images of TUNEL staining showing cardiac cell apoptosis; $n=10$. (B) The percentage of apoptotic cells is shown; $n=10$. (C) The protein levels of apoptosis related factors in mice at 3 days after AMI; $n=8$. (D) The protein levels were quantified by densitometric analysis and normalized to levels of $\beta$-tubulin; $\mathrm{n}=8$. (E) Ratio of bax $/ \mathrm{bcl}-2 ; \mathrm{n}=8$. ${ }^{* *} P<0.01$.

Mirt1 is mainly expressed in cardiac fibroblasts

To confirm the localization of Mirt1 in the myocardium, we isolated cardiomyocytes and CFs from the hearts of mice subjected to coronary artery ligation and from the sham group. The basal level of Mirt1 in CFs was approximately 7-fold higher than that in cardiomyocytes, and there was no significant difference in the expression of Mirt1 in cardiomyocytes between the operation group and the sham group (Fig. 1B). However, the level of Mirt1 in the CFs of infarcted myocardium was markedly higher than that of the sham group, showing an increase of almost 9-fold (Fig. 1B).

\section{The effects of knockdown of Mirt1 on heart functions and myocardial infarct size}

We established an adenovirus-mediated shRNA against Mirt1 (Ad-shMirt1). Mice were injected with Ad-shMirt1 or Ad-shCon (Adenovirus-mediated control shRNA vector) through intramyocardial injection 7 days before myocardial infarction surgery (Fig. 1C). Compared with Ad-shCon treated mice, the expression of Mirt1 in Ad-shMirt1 treated mice decreased at 24 hours in both the AMI and the sham group (Fig. 2A). Cardiac functions were evaluated at 3 days after operation using transthoracic echocardiography and hemodynamic analysis. Compared with the sham group, cardiac functions, assessed by left ventricle ejection fraction (LVEF), left ventricle fractional shortening (LVFS), diastolic and systolic left ventricular internal diameter (LVID,d, LVID,s) left ventricular (LV) maximum pressure and $\mathrm{dP} / \mathrm{dt}$ maximum rate in AMI mice were obviously impaired. However, knockdown of Mirt1 significantly improved cardiac functions of AMI mice at 3 days, but had no effect in the sham group (Fig. 2B, C, D). As shown in Fig. 2E, Evans blue and TTC staining demonstrated that the myocardial infarct size of AMI mice treated with Ad-shMirt1 was decreased by about $30 \%$, compared with mice receiving Ad-shCon. However, there was no significant difference in 


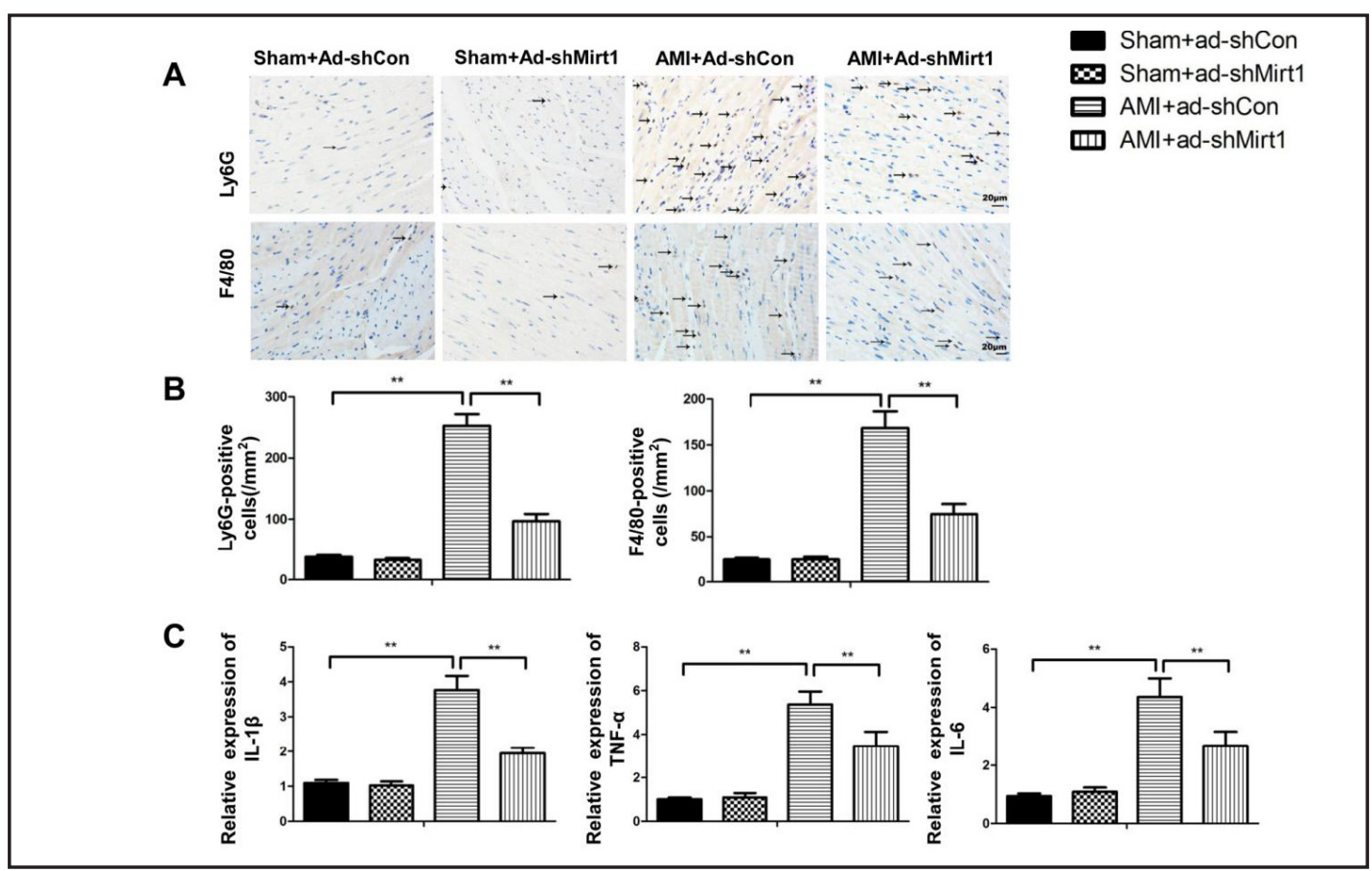

Fig. 4. Knockdown of Mirt1 inhibits inflammatory cells infiltration in vivo. (A) Representative images of immunohistochemical staining for Ly6G+ (neutrophil) and F4/80+ (macrophages) in infarcted myocardium at 3 days after AMI; $\mathrm{n}=8$. (B) The number of Ly6G+ and F4/80+ cells per square millimeter $\left(\mathrm{mm}^{2}\right)$ of heart sections; $\mathrm{n}=8$. (C) The mRNA expression of IL-1 $\beta$, TNF- $\alpha$ and IL- 6 in infarcted myocardium; $\mathrm{n}=10 .{ }^{* *} P<0.01$.

areas at risk between the two groups. These data reveal that inhibition of Mirt1 expression improves the cardiac functions of AMI mice.

Knockdown of Mirt1 inhibits apoptosis and decreases the inflammatory cell infiltration after AMI in the infarcted myocardium

To further clarify the mechanism of the protective effects of Ad-shMirt1 in AMI mice, cell apoptosis was assessed by TUNEL immunofluorescence staining. As shown in Fig. 3A, the number of TUNEL-positive cells in the infarcted area of the Ad-shMirt1 treated mice was significantly decreased, compared with that of Ad-shCon group. We also analyzed the protein levels of cleaved caspase-3, Bax, and Bcl-2 by western blotting (Fig. 3C) .The expression of cleaved caspase- 3 was inhibited and the ratio of Bax/Bcl-2 was decreased in the AMI mice treated with Ad-shMirt1 compared with those treated with Ad-shCon (Fig. 3D, E). These data indicate that Mirt1 modulates myocardial apoptosis, at least in part, through regulation of the activation of caspase- 3 and the ratio of pro-apoptotic Bax to anti-apoptotic Bcl-2. As immunohistochemical staining demonstrated, the number of inflammatory cells (neutrophils and macrophages) infiltrating into the myocardium of Ad-shMirt1 treated mice was significantly decreased after AMI, compared with that of Ad-shCon treated mice (Fig. 4A, B). Compared to Ad-shCon treated mice, the mRNA levels of inflammatory factors, including interleukin $-1 \beta$ (IL-1 $\beta$ ), tumor necrosis factor- $\alpha$ (TNF- $\alpha$ ) and interleukin-6 (IL-6) were decreased in Ad-shMirt1 treated mice after AMI (Fig. 4C). These results demonstrate that the inhibition of Ad-shMirt1 in inflammatory cell migration may be mediated through suppression of the expression of inflammatory factors.

Knockdown of Mirt1 in cardiac fibroblasts inhibits cardiomyocytes apoptosis and decreases peritoneal macrophage migration in vitro

Cardiac fibroblasts were isolated from new born C57 mice (nCFs). The expression of Mirt1 in nCFs was significantly increased at 6 hours under hypoxic conditions, when it 
A
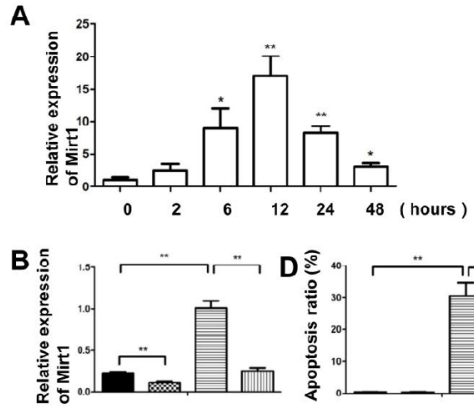

E

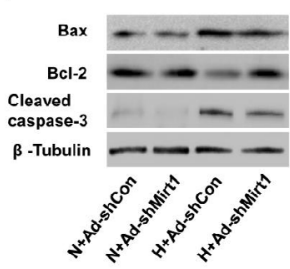

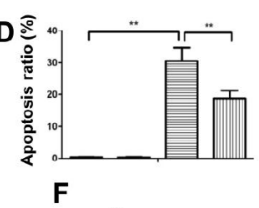

$\mathbf{F}$

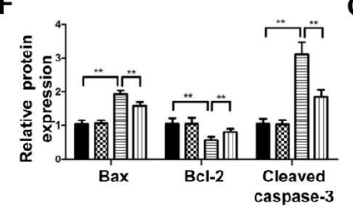

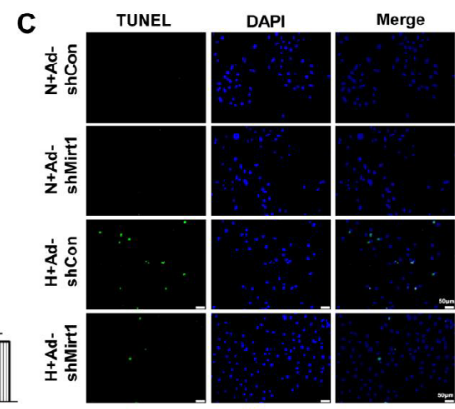

$\mathbf{G}$

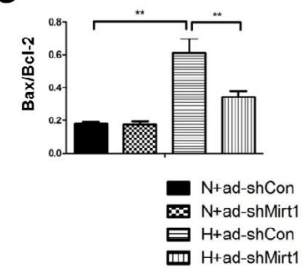

Fig. 5. Knock down of Mirt1 in nCFs suppresses the apoptosis of cardiomycytes in vitro. (A) The expression of Mirt1 in CFs cultured under hypoxia for $0,2,6,12,24$ and 48 hours. ${ }^{*} P<0.05,{ }^{* *} P<0.01$ versus the values at 0 hours. (B) The efficiency of Ad-shMirt1 in nCFs cultured under hypoxia (H) or normoxia (N) for 12 hours. (C, D) The apoptosis of cardiomycytes co-cultured with nCFs infected with Ad-shMirt1 or Ad-shCon and cultured under hypoxic or normoxic conditions for 12 hours. (E) The protein levels of apoptosis related factors of cardiomyocytes co-cultured with nCFs infected with Ad-shMirt1 or Ad-shCon and cultured under hypoxia or normoxia for 12 hours. (F) The protein levels were quantified by densitometric analysis and normalized to levels of $\beta$-tubulin. (G) Ratio of bax/bcl-2. Data are representative of three independent experiments and expressed as the mean $\pm \mathrm{SD},{ }^{*} P<0.05,{ }^{* *} P<0.01$.

was approximately 9-fold higher than controls, peaked at 12 hours, at about 18 -fold higher than controls, and decreased to a normal level at 48 hours (Fig. 5A). Ad-shMirt1 effectively inhibited the expression of Mirt1 in nCFs suffered from cultured under hypoxic or normoxic conditions (Fig. 5B). The cardiomyocytes isolated from new born mice were co-cultured with nCFs infected with Ad-shMirt1 or Ad-shCon and then subjected to hypoxia or normoxia for 12 hours. Under hypoxic conditions, the apoptosis rate of cardiomyocytes co-cultured with nCFs infected with Ad-shMirt1 was reduced, compared with those infected with Ad-shCon (Fig. 5C, D). The results indicated that inhibition of the expression of Mirt1 in nCFs reduced the apoptosis of cardiomyocytes under hypoxic conditions. Furthermore, western blotting also showed that the level of cleaved caspase- 3 was inhibited and the ratio of Bax/Bcl-2 was decreased in cardiomyocytes co-cultured with nCFs infected with Ad-shMirt1 under hypoxic conditions, results which were consistent with our in vivo experiments (Fig. 5E, F).

After hypoxic or normoxic culture for 12 hours, the conditioned medium of nCFs pretreated with Ad-shMirt1 or Ad-shCon was collected and added to the lower chambers of the coculture system, and primary peritoneal macrophages were added to the upper chambers. The results showed that conditioned medium from supernatant of hypoxic nCFs pretreated with Ad-shMirt1 remarkably inhibited the migration of peritoneal macrophages cultured in the upper chambers (Fig. 6A, B). Moreover, we measured the levels of inflammatory cytokines in the supernatant of nCFs in the four groups. Hypoxic culture markedly increased the concentration of IL- $1 \beta$, TNF- $\alpha$ and IL- 6 in the supernatant. However, the hypoxia-induced upregulation of IL-1 $\beta$, TNF- $\alpha$ and IL- 6 in nCFs was significantly inhibited by Ad-shMirt1. No significant difference was observed between the two groups under normoxic conditions (Fig. 6C). 


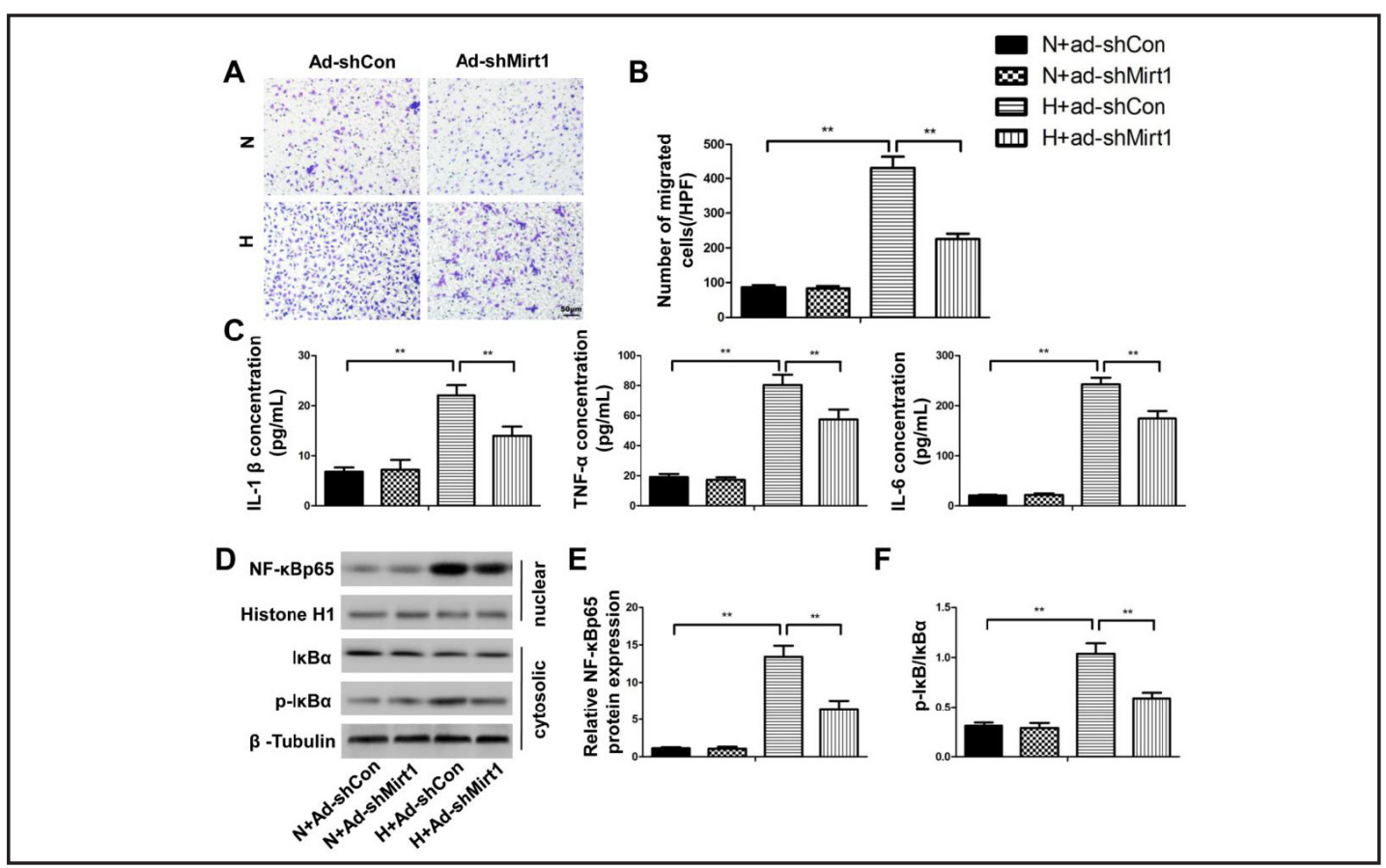

Fig. 6. Knock down of Mirt1 in nCFs suppresses the migration of peritoneal macrophages and nuclear translocation of NF- $\mathrm{KB}$ under hypoxia in vitro. (A, B) The migration of peritoneal macrophages co-cultured with conditioned medium from nCFs infected with Ad-shMirt1 or Ad-shCon under hypoxic $(\mathrm{H})$ or normoxic (N) conditions for 12 hours. HPF indicates high-power field. (C) The concentrations of IL-1 $\beta$, TNF- $\alpha$ and IL-6 in the supernatant of nCFs infected with Ad-shMirt1 or Ad-shCon and cultured under hypoxia or normoxia for 12 hours. (D) Protein was extracted from the nuclei and cytosol of the four groups of nCFs. The protein

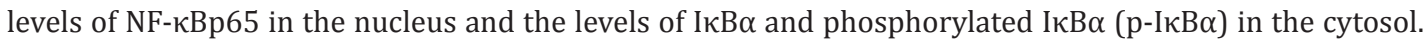
(E) The NF- $\kappa B p 65$ levels were quantified by densitometric analysis normalized to levels of histone H1. (F) Ratio of $\mathrm{p}-\mathrm{I} \kappa \mathrm{B} \alpha / \mathrm{I} \kappa \mathrm{B} \alpha$. Data shown are representative of three independent experiments and are expressed as the mean \pm SD. ${ }^{* *} P<0.01$.

Knockdown of Mirt1 in cardiac fibroblasts inhibits NF- $\kappa B$ activation under hypoxic conditions

AMI promotes the expression of NF- $\kappa B$ dependent inflammatory genens in infarcted myocardium. We analyzed the levels of p65, a major subunit of NF- $\mathrm{KB}$, in nuclear extract of nCFs treated with Ad-shMirt1 or Ad-Con, under either hypoxic or normoxic culture conditions for 12 hours. The nuclear NF- $\mathrm{kB}$ subunit p65 was significantly increased in the hypoxia group compared with normoxic cultured cells, and was inhibited in the nCFs treated with Ad-shMirt1 under hypoxia (Fig. 6D, E). Phosphorylation-dependent degradation of $\mathrm{I} \kappa \mathrm{B} \alpha$ in the cytosol is required for nuclear translocation of NF- $\kappa \mathrm{B}$. The results demonstrated an increased level of phosphorylated IкB $\alpha$ in hypoxia, which was suppressed by Ad-shMirt1 in nCFs under hypoxic conditions (Fig. 6D, F). These data indicated that knockdown of Mirt1 suppressed NF- $\mathrm{KB}$ activation in hypoxic nCFs

\section{Discussion}

LncRNAs are a novel class of non-protein coding transcripts involved in a variety of physiological and pathological processes [22,23]. Hundreds of IncRNAs have been reported to play important roles in cardiovascular diseases, particularly in ischemic cardiomyopathy $[24,25]$. Our study demonstrated the functions of the novel lncRNA Mirt1 identified in a previous study [11] in AMI mice. The major findings were: 1) Mirt1 was mainly expressed in cardiac fibroblasts, rather than cardiomyocytes; 2) knockdown of Mirt1 improved cardiac 
functions, and reduced infarct size in AMI mice; 3) Mirt1 expression in cardiac fibroblasts could be induced by hypoxia in vitro; 4) inhibiting the expression of Mirt1 in CFs alleviated the apoptosis of myocardium both in mice and in cultured cardiomyocytes; 5) restraining the expression of Mirt1 inhibited inflammatory cell infiltration in vivo and in vitro; 6) knockdown of Mirt1 repressed the nuclear translocation of NF- $\kappa B$ in CFs under hypoxia.

Apoptosis of cardiomyocytes is crucial in the pathogenesis of AMI [26, 27], and could determine the fate of the heart [28]. In our present study, the myocardium of AMI mice treated with Ad-shMirt1exhibited a lower rate of apoptosis. Accordingly, cardiomyocytes were cocultured under hypoxic conditions with fibroblasts treated with Ad-shMirt1 or Ad-shCon. Consistent with our in vivo experiments, apoptosis of the cardiomyocytes in the Ad-shMirt1 group was also inhibited. We speculated that cardiomyocyte apoptosis could be affected by some kind of apoptosis related factors regulated by Mirt1 in fibroblasts.

It is generally known that cardiac fibroblasts play a key role in repair and remodeling of the heart after myocardial infarction, and an increasing number of studies have focused on the autocrine or paracrine effects of cytokines secreted by cardiac fibroblasts during AMI. These factors mainly include proinflammatory cytokines (e.g. TNF $\alpha$, IL-1 $\beta$ and IL-6) [29-31], chemokines (e.g.ICAM-1, E-selectin and CXCs) [6], profibrotic factors and factors regulating neovascularization. Previous studies have demonstrated the important role of cardiac fibroblasts in inflammatory responses and subsequent injury after myocardial infarction [4]. In this study, we discovered that inhibiting expression of Mirt1 was able to repress inflammatory cell infiltration into the myocardium of AMI mice, and that knockdown of Mirt1 in hypoxic nCFs significantly inhibited the migration of macrophages in vitro. Hence, we speculated that Mirt1 may modulate the secretion of some cytokines or chemokines in CFs, which could affect inflammatory cell infiltration or migration. We measured the expression of proinflammatory cytokines released by CFs, and observed that the expression of TNF $\alpha$, IL-1 $\beta$ and IL- 6 were significantly inhibited by Ad-shMirt1 both in the hearts of AMI mice and in CFs under hypoxic conditions. In addition, these proinflammatory cytokines have also been proven to have effects on cardiomyocyte apoptosis [32, 33]. We speculate that the protective effects of Ad-shMirt1 may be associated with suppression of the levels of these proinflammatory cytokines

Activation of NF- $\mathrm{KB}$ is essential in initiating the inflammatory response in myocardial injury. A series of studies have demonstrated that blockage of NF- $\kappa B$ could protect the heart against acute ischemic injury and inflammatory response [34]. NF- $\mathrm{BB}$ is localized in the cytoplasm in a complex with inhibitory proteins (IкBs) under normal conditions. Many stimuli promote the site-specific phosphorylation and proteosomal degradation of IкBs, resulting in, NF- $\kappa B$ nuclear translocation, DNA binding, and transcription of specific genes. In this study we showed that inhibition of Mirt1 expression could suppress the phosphorylation

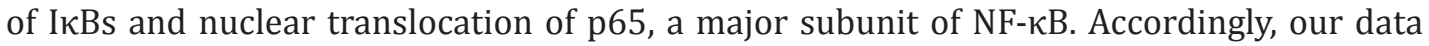
suggest that the protective effects of Mirt1 knockdown in AMI may be due to the inhibition of NF- $\kappa B$ activation, thus decreasing the levels of proinflammatory cytokines which could mediate the inflammatory response and promote cell apoptosis.

This study has some limitations as follows: 1 ) the effects of Mirt1 overexpression in cardiac fibroblasts were not elaborated; 2) the mechanisms involved in NF- $\kappa$ B inhibition mediated by Mirt1 knockdown were not clear, and will require further study. 3) in viewof the fact that the human homolog of murine Mirt1 has not been identified, its potential for use in treating human diseases currently seems relatively limited.

\section{Acknowledgements}

This research did not receive any specific grant from funding agencies in the public, commercial, or not-for-profit sectors. 


\section{Cellular Physiology Cell Physiol Biochem 2017;42:1153-1164 and Biochemistry Publis.1159/000478870 $2017 \quad \begin{aligned} & \text { DOI: } 2017 \text { The Author(s). Published by S. Karger AG, Basel } \\ & \text { www.karger.com/cpb }\end{aligned}$}

Li/Zhou/Huang: Inhibition of Mirt1 Attenuates Acute Myocardial Infarction

\section{Disclosure Statement}

None.

\section{References}

1 Mozaffarian D, Benjamin EJ, Go AS, Arnett DK, Blaha MJ, Cushman M, de Ferranti S, Despres JP, Fullerton HJ, Howard VJ, Huffman MD, Judd SE, Kissela BM, Lackland DT, Lichtman JH, Lisabeth LD, Liu S, Mackey RH, Matchar DB, McGuire DK, Mohler ER, 3rd, Moy CS, Muntner P, Mussolino ME, Nasir K, Neumar RW, Nichol G, Palaniappan L, Pandey DK, Reeves MJ, Rodriguez CJ, Sorlie PD, Stein J, Towfighi A, Turan TN, Virani SS, Willey JZ, Woo D, Yeh RW, Turner MB: Heart disease and stroke statistics--2015 update: a report from the American Heart Association. Circulation 2015;131:e29-322.

-2 Marchant DJ, Boyd JH, Lin DC, Granville DJ, Garmaroudi FS, McManus BM: Inflammation in myocardial diseases. Circ Res 2012;110:126-144.

-3 Konstantinidis K, Whelan RS, Kitsis RN: Mechanisms of cell death in heart disease. Arterioscler Thromb Vasc Biol 2012;32:1552-1562.

-4 Kawaguchi M, Takahashi M, Hata T, Kashima Y, Usui F, Morimoto H, Izawa A, Takahashi Y, Masumoto J, Koyama J, Hongo M, Noda T, Nakayama J, Sagara J, Taniguchi S, Ikeda U: Inflammasome activation of cardiac fibroblasts is essential for myocardial ischemia/reperfusion injury. Circulation 2011;123:594-604.

5 Saxena A, Chen W, Su Y, Rai V, Uche OU, Li N, Frangogiannis NG: IL-1 induces proinflammatory leukocyte infiltration and regulates fibroblast phenotype in the infarcted myocardium. J Immunol 2013;191:48384848.

6 Turner NA, Das A, O'Regan DJ, Ball SG, Porter KE: Human cardiac fibroblasts express ICAM-1, E-selectin and CXC chemokines in response to proinflammatory cytokine stimulation. Int J Biochem Cell Biol 2011;43:1450-1458.

7 Uchida S, Dimmeler S: Long noncoding RNAs in cardiovascular diseases. Circ Res 2015;116:737-750.

-8 Vausort M, Wagner DR, Devaux Y: Long noncoding RNAs in patients with acute myocardial infarction. Circ Res 2014;115:668-677.

9 Liu L, An X, Li Z, Song Y, Li L, Zuo S, Liu N, Yang G, Wang H, Cheng X, Zhang Y, Yang X, Wang J: The H19 long noncoding RNA is a novel negative regulator of cardiomyocyte hypertrophy. Cardiovasc Res 2016;111:5665.

10 Wu H, Liu J, Li W, Liu G, Li Z: LncRNA-HOTAIR promotes TNF-alpha production in cardiomyocytes of LPSinduced sepsis mice by activating NF-kappaB pathway. Biochem Biophys Res Commun 2016;471:240-246.

11 Zangrando J, Zhang L, Vausort M, Maskali F, Marie PY, Wagner DR, Devaux Y: Identification of candidate long non-coding RNAs in response to myocardial infarction. BMC Genomics 2014;15:

12 Tang Y, Wang Y, Park KM, Hu Q Teoh JP, Broskova Z, Ranganathan P, Jayakumar C, Li J, Su H, Ramesh G, Kim IM: MicroRNA-150 protects the mouse heart from ischaemic injury by regulating cell death. Cardiovasc Res 2015;106:387-397.

13 Jiang Y, Bai J, Tang L, Zhang P, Pu J: Anti-CCL21 antibody attenuates infarct size and improves cardiac remodeling after myocardial infarction. Cell Physiol Biochem 2015;37:979-990.

-14 Du M, Huang K, Huang D, Yang L, Gao L, Wang X, Li X, Wang C, Zhang F, Wang Y, Cheng M, Tong Q, Qin G, Wang L: Renalase is a novel target gene of hypoxia-inducible factor-1 in protection against cardiac ischaemia-reperfusion injury. Cardiovasc Res 2015;105:182-191.

15 Luo G, Li Q, Zhang X, Shen L, Xie J, Zhang J, Kitakaze M, Huang X, Liao Y: Ablation of C/EBP homologous protein increases the acute phase mortality and doesn't attenuate cardiac remodeling in mice with myocardial infarction. Biochem Biophys Res Commun 2015;464:201-207.

16 Gao E, Lei YH, Shang X, Huang ZM, Zuo L, Boucher M, Fan Q Chuprun JK, Ma XL, Koch WJ: A novel and efficient model of coronary artery ligation and myocardial infarction in the mouse. Circ Res 2010;107:1445-1453.

17 O'Connell TD, Swigart PM, Rodrigo MC, Ishizaka S, Joho S, Turnbull L, Tecott LH, Baker AJ, Foster E, Grossman W, Simpson PC: Alpha1-adrenergic receptors prevent a maladaptive cardiac response to pressure overload. J Clin Invest 2006;116:1005-1015. 


\section{Cellular Physiology Cell Physiol Biochem 2017;42:1153-1164 \begin{tabular}{l|l} 
DOI: 10.1159/000478870 & $\begin{array}{l}\text { O 2017 The Author(s). Published by S. Karger AG, Basel } \\
\text { www.karger.com/cpb }\end{array}$
\end{tabular}}

Li/Zhou/Huang: Inhibition of Mirt1 Attenuates Acute Myocardial Infarction

18 Huang ZP, Chen J, Seok HY, Zhang Z, Kataoka M, Hu X, Wang DZ: MicroRNA-22 regulates cardiac hypertrophy and remodeling in response to stress. Circ Res 2013;112:1234-1243.

19 Ehler E, Moore-Morris T, Lange S: Isolation and culture of neonatal mouse cardiomyocytes. J Vis Exp 2013;10.3791/50154

-20 Pineda-Torra I, Gage M, de Juan A, Pello OM: Isolation, Culture, and Polarization of Murine Bone MarrowDerived and Peritoneal Macrophages. Methods Mol Biol 2015;1339:101-109.

-21 Zoccarato A, Surdo NC, Aronsen JM, Fields LA, Mancuso L, Dodoni G, Stangherlin A, Livie C, Jiang H, Sin YY, Gesellchen F, Terrin A, Baillie GS, Nicklin SA, Graham D, Szabo-Fresnais N, Krall J, Vandeput F, Movsesian M, Furlan L, Corsetti V, Hamilton G, Lefkimmiatis K, Sjaastad I, Zaccolo M: Cardiac Hypertrophy Is Inhibited by a Local Pool of cAMP Regulated by Phosphodiesterase 2. Circ Res 2015;117:707-719. Ponting CP, Oliver PL, Reik W: Evolution and functions of long noncoding RNAs. Cell 2009;136:629-641. Wang KC, Chang HY: Molecular mechanisms of long noncoding RNAs. Mol Cell 2011;43:904-914. Archer K, Broskova Z, Bayoumi AS, Teoh JP, Davila A, Tang Y, Su H, Kim IM: Long Non-Coding RNAs as Master Regulators in Cardiovascular Diseases. Int J Mol Sci 2015;16:23651-23667.

25 Ounzain S, Burdet F, Ibberson M, Pedrazzini T: Discovery and functional characterization of cardiovascular long noncoding RNAs. J Mol Cell Cardiol 2015;89:17-26.

26 Liu Y, Zhou D, Li G, Ming X, Tu Y, Tian J, Lu H, Yu B: Long non coding RNA-UCA1 contributes to cardiomyocyte apoptosis by suppression of p27 expression. Cell Physiol Biochem 2015;35:1986-1998.

-27 Zhang J, He Z, Xiao W, Na Q Wu T, Su K, Cui X: Overexpression of BAG3 Attenuates Hypoxia-Induced Cardiomyocyte Apoptosis by Inducing Autophagy. Cell Physiol Biochem 2016;39:491-500.

-28 Krijnen PA, Nijmeijer R, Meijer CJ, Visser CA, Hack CE, Niessen HW: Apoptosis in myocardial ischaemia and infarction. J Clin Pathol 2002;55:801-811.

29 Burger A, Benicke M, Deten A, Zimmer HG: Catecholamines stimulate interleukin-6 synthesis in rat cardiac fibroblasts. Am J Physiol Heart Circ Physiol 2001;281:H14-21.

-30 Cowling RT, Gurantz D, Peng J, Dillmann WH, Greenberg BH: Transcription factor NF-kappa B is necessary for up-regulation of type 1 angiotensin II receptor mRNA in rat cardiac fibroblasts treated with tumor necrosis factor-alpha or interleukin-1 beta. J Biol Chem 2002;277:5719-5724.

31 Lin CC, Yang CC, Wang CY, Tseng HC, Pan CS, Hsiao LD, Yang CM: NADPH Oxidase/ROS-Dependent VCAM1 Induction on TNF-alpha-Challenged Human Cardiac Fibroblasts Enhances Monocyte Adhesion. Front Pharmacol 2015;6:310.

-32 Cao YY, Chen ZW, Gao YH, Wang XX, Ma JY, Chang SF, Qian JY, Ge JB: Exenatide Reduces Tumor Necrosis Factor-alpha-induced Apoptosis in Cardiomyocytes by Alleviating Mitochondrial Dysfunction. Chin Med J (Engl) 2015;128:3211-3218.

-33 Okada M, Yamawaki H: Levosimendan inhibits interleukin-1beta-induced apoptosis through activation of Akt and inhibition of inducible nitric oxide synthase in rat cardiac fibroblasts. Eur J Pharmacol 2015;769:86-92.

34 Nishikido T, Oyama J, Shiraki A, Komoda H, Node K: Deletion of Apoptosis Inhibitor of Macrophage (AIM)/ CD5L Attenuates the Inflammatory Response and Infarct Size in Acute Myocardial Infarction. J Am Heart Assoc 2016;5:e002863. 